\title{
Interstitial lung disease in amyopathic dermatomyositis, dermatomyositis and polymyositis
}

\author{
V. Cottin*, F. Thivolet-Béjui\#, M. Reynaud-Gaubert ${ }^{+}$, J. Cadranel ${ }^{\S}$, P. Delaval ${ }^{f}$, P-J. Ternamian", \\ J-F. Cordier* and the Groupe d'Etudes et de Recherche sur les Maladies "Orphelines" Pulmonaires \\ (GERM"O"P)
}

Interstitial lung disease in amyopathic dermatomyositis, dermatomyositis and polymyositis. V. Cottin, F. Thivolet-Béjui, M. Reynaud-Gaubert, J. Cadranel, P. Delaval, P-J. Ternamian, J-F. Cordier and the Groupe d'Etudes et de Recherche sur les Maladies "Orphelines" Pulmonaires (GERM"O"P). (C) ERS Journals Ltd 2003.

ABSTRACT: This study investigated interstitial pneumonia associated with amyopathic dermatomyositis, dermatomyositis and polymyositis, paying particular attention to muscular and/or cutaneous manifestations and their chronology relative to lung involvement. Patients included four males and 13 females, aged $51.7 \pm 10.8$ yrs, who had surgical lung biopsy.

Diagnoses included dermatomyositis (10 patients), polymyositis (four patients) and amyopathic dermatomyositis (three patients). Solitary respiratory manifestations preceded the onset of any skin or muscle disease in four cases $(24 \%)$. Reticular and ground glass opacities were the most frequent computed tomography (CT) findings. Pathological review showed nonspecific interstitial pneumonia (eleven, 65\%; cellular, two; cellular and fibrotic, five; fibrotic, four), usual interstitial pneumonia (two), organising pneumonia (two), lymphocytic interstitial pneumonia (one), and unclassifiable interstitial pneumonia (one). Nonspecific interstitial pneumonia was the most common histological pattern of interstitial pneumonia in patients with amyopathic dermatomyositis (three of three) and in patients with respiratory symptoms as the initial clinical manifestation of the connective tissue disease (three of four). Survival at 5 yrs was $50 \%$.

This study shows the clinician should remain alert to potential muscular or cutaneous manifestations whenever a pathological diagnosis of nonspecific interstitial pneumonia is made.

Eur Respir J 2003; 22: 245-250.
*Dept of Respiratory Medicine, "Dept of Pathology, "Dept of Radiology, Louis Pradel Hospital, Claude Bernard University, Lyon, ${ }^{+}$Dept of Thoracic Surgery, Sainte-Marguerite Hospital, Marseille, ${ }^{\S}$ Dept of Respiratory Medicine, Tenon Hospital, Paris, and ${ }^{f}$ Dept of Respiratory and Cardiology Medicine, Pontchaillou Hospital, Rennes, France.

Correspondence: J-F. Cordier, Groupe d'Etudes et de Recherche sur les Maladies "Orphelines" Pulmonaires, Hôpital Cardiovasculaire et Pneumologique Louis Pradel, 69394 Lyon Cedex 03, France.

Fax: 33472357653

E-mail: germop@univ-lyonl.fr

Keywords: Dermatomyositis, fibrosis, interstitial pneumonia, nonspecific

Received: March 102003

Accepted after revision: April 222003

This work was supported by a grant from Hospices Civils de Lyon, Programme Hospitalier de Recherche Clinique, France (93.97005).
Interstitial lung disease is now recognised as a direct manifestation of dermatomyositis/polymyositis (DPM), present on the chest radiograph in $7 \%$ of patients [1], and associated with a poor outcome [2]. The histopathological patterns described in patients with DPM-associated interstitial lung disease include bronchiolitis obliterans organising pneumonia, usual interstitial pneumonia (UIP), and diffuse alveolar damage [3-6]. However, most published series preceded the recognition of idiopathic nonspecific interstitial pneumonia (NSIP) [7], which has recently been reported in DPM [7, 8], and may represent the most common histological pattern of DPM-associated interstitial pneumonia [1].

Although DPM often includes both muscle and skin involvement [9], these may be dissociated. Florid cutaneous manifestations of DPM may occasionally be present in a patient for a prolonged period of time without any clinical evidence of underlying myositis [10], a condition termed "amyopathic dermatomyositis", or "dermatomyositis sine myositis". Thus, DPM is considered as a clinical spectrum, including polymyositis, dermatomyositis and amyopathic dermatomyositis [11]. In addition, interstitial pneumonia associated with DPM may precede by several months the cutaneous and/or muscular symptoms of the disease [3, 4].

Diagnostic criteria by BOHAN and PETER [9] for DPM have been used in most studies. Since muscle disease is a required criterion, little is known about lung involvement in patients with amyopathic dermatomyositis. Whether the lung involvement is similar in dermatomyositis, polymyositis and amyopathic dermatomyositis, has not been addressed specifically. Hence, patients with amyopathic dermatomyositis were not included in two recent studies [1, 12].

This study was undertaken to characterise the clinical and histopathological features of interstitial pneumonia associated with DPM, with particular attention to the muscular and/or cutaneous manifestations of the disease, and of their chronology relative to lung involvement.

\section{Patients and methods}

\section{Case recruitment}

This retrospective multicentre study was conducted by the Groupe d'Etudes et de Recherche sur les Maladies "Orphelines" Pulmonaires (GERM"O"P). A letter was sent to all participating physicians asking them to report all known cases of patients with DPM-associated interstitial lung 
disease, in whom a lung biopsy had been performed between 1989-2001.

\section{Inclusion criteria}

Cases were acceptable for inclusion in the study if they fulfilled the following criteria. 1) Criteria for the diagnosis of DPM according to BOHAN and PETER [9], namely: symmetrical proximal muscle weakness, elevated muscle enzymes in serum, suggestive electromyographic abnormalities, muscle biopsy changes, and characteristic skin rash of dermatomyositis. The diagnosis was considered definite, probable, or possible, according to the number of fulfilled criteria (at least four, three, or two, respectively, including the skin rash for the diagnosis of dermatomyositis). In addition, the diagnosis of amyopathic dermatomyositis was made on the basis of characteristic manifestations of dermatomyositis, without clinical evidence of muscle disease [10]. 2) Presence of diffuse infiltrative lung disease on CT of the chest. 3) Open or videothoracoscopic surgical lung biopsy.

\section{Data collection}

The clinical data were collected through a detailed questionnaire that was sent to all participating physicians. High-resolution computed tomography scans of the chest were reviewed separately by two of the authors (V. Cottin, P-J. Ternamian) without knowledge of the pathological diagnosis. Consensus data were collected for the main interstitial abnormalities, as defined elsewhere [13]. Following the assessment of the images, the observers were asked which pattern of idiopathic interstitial pneumonia would be best suggested by the CT findings on the basis of published data.

\section{Pathological review}

Pathological material was retrospectively reviewed by French pathologists expert in the field of interstitial lung disease, including one of the authors. In addition, the pathological diagnosis of NSIP was confirmed by a panel of pathologists of the American Thoracic Society workshop on NSIP in two patients (cases number six and 14) in whom the interstitial lung disease preceded the onset of DPM. The slides were reviewed without knowledge of clinical or radiological information, and classified as defined elsewhere [14]. Data are presented as mean $\pm \mathrm{SD}$.

\section{Results}

\section{Patients}

In total, 17 completed questionnaires were received for analysis. Patient number six had participated in a previous study [8]. The patients included four males and 13 females, aged $51.7 \pm 10.8$ yrs. They were current smokers in one case, former smokers in three cases and 11 patients had never smoked (the information was not available for two patients).

\section{Features of the muscle and skin disease}

The diagnoses separated as follows (table 1): dermatomyositis, 10 of 17 cases; polymyositis, four of 17 cases; and amyopathic dermatomyositis, three of 17 cases. The confidence for the diagnosis of DPM was definite in eight cases, and probable in six cases.

Twelve patients had symmetrical proximal muscle weakness (table 2), responsible for limitation of daily activity in nine cases. Electromyogram performed in 14 cases contributed to the diagnosis of myositis in nine cases. Skeletal muscle biopsy, performed in 15 cases, contributed to the diagnosis in 11 cases. A skin biopsy was performed in 12 patients, and suggested the diagnosis of DPM in six patients.

In the three patients with amyopathic dermatomyositis, the diagnosis was established on the presence of a skin rash characteristic of DPM, associated with elevated muscle enzymes. No muscle weakness occurred during follow-up (6.8 yrs in patient number one, 2 yrs in patients number two and three). Muscle biopsy and electromyogram were not contributive (patient number three) or not performed (patients number one and two). The skin manifestations in patients with amyopathic dermatomyositis included the following: 1) skin rash localised to the face and upper anterior chest (patient number three); 2) violaceous erythema and oedema of eyelids and periorbital tissue (heliotrope erythema), rash of the dorsal side of the interphalangeal joints of the hands and feet, and erythema of the dorsal side of the elbows (patient number one); and 3) Gottron's sign of the dorsal aspect of the forearms and elbows, with erythema of the upper chest and of the periorbital areas, and poikiloderma of the palms (patient number two).

\section{Lung pathology}

An open ( $n=7)$ or video-assisted thoracoscopic $(n=11)$ lung biopsy was available in all cases. Five biopsies were performed before 1994. The patients had not received corticosteroid treatment at the time of the biopsy. After pathological review, the final pathological diagnosis was UIP in two cases (table 1). In four cases, the diagnosis of UIP initially made was changed to NSIP after review. The most common diagnosis was NSIP in 11 of 17 cases $(65 \%)$, including two NSIP group I (cellular NSIP), five NSIP group II (both inflammatory and fibrotic lesions), and four NSIP group III (predominantly fibrotic) [7]; fibrosing NSIP (including subgroups II and III) accounted for $82 \%$ of NSIP. The other pathological diagnoses consisted of organising pneumonia (two cases), and lymphoid interstitial pneumonia (one case). In addition, one case could not be classified despite an adequately sized video-assisted lung biopsy $(5 \times 15 \mathrm{~mm})$, and extensive re-examinations, and was therefore diagnosed as unclassifiable interstitial pneumonia.

\section{Respective chronology of pulmonary, cutaneous and muscular manifestations}

The mean interval between the onset of respiratory symptoms and the pathological diagnosis was $126 \pm 96$ days (range 25-352 days). Pulmonary symptoms preceded the onset of cutaneous symptoms in three cases (median interval, 100 days), and occurred after the skin manifestations in three cases (median interval, 90 days). Pulmonary symptoms preceded muscular manifestations in four patients (median interval, 341 days) and occurred after the onset of the muscle disease in two patients. Muscular or dermal manifestations were still present at the time of the diagnosis of lung disease when they occurred before the diagnosis of lung involvement, although the symptoms had improved with treatment. The onset of pulmonary symptoms was concomitant of cutaneous and muscular symptoms in seven and eight cases, respectively. 


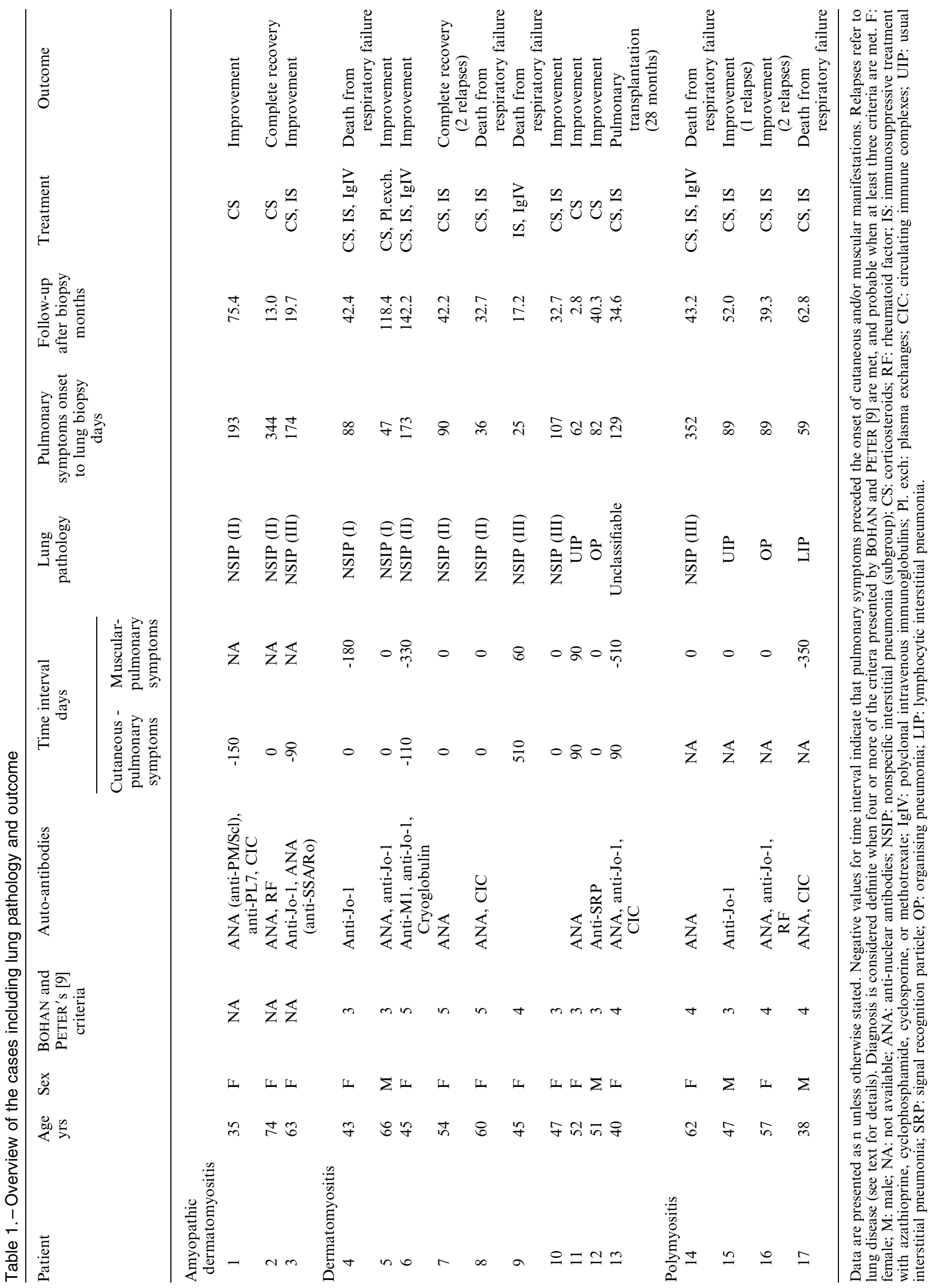


Table 2.-Cutaneous and musculoskeletal manifestations

Patients

Muscle symptoms

Proximal muscle weakness

$12 / 17(71)$

Muscle pain, tenderness

$14 / 17(82)$

Muscle swelling

Muscle atrophy

2/17 (12)

$4 / 17$ (24)

$13 / 17(76)$

$4 / 17(24)$

Arthritis

$7 / 17(41)$

$13 / 17(76)$

$7 / 17(41)$

$11 / 13(85)$

$4 / 15(27)$

$9 / 12(75)$

$9 / 13(69)$

Elevated aldolase level

Elevated creatine phosphokinase level

Data are presented as $\mathrm{n} /$ total $\mathrm{n}(\%)$.

Interestingly, solitary pulmonary manifestations preceded any skin or muscle disease in four of 17 patients, including three with a histopathology of NSIP (table 1). In patient number six, the respiratory disease preceded the onset of cutaneous and muscular manifestations, which appeared after the lung disease had resolved under treatment. A relapse of DPM occurred 4 yrs later, with skin and muscle manifestations, but there was no relapse of lung disease.

\section{Clinical and functional respiratory manifestations}

Most patients presented with asthenia, fever, and/or weight loss (mean weight loss $10.1 \pm 5.6 \mathrm{~kg}$ ), and dyspnoea. Pulmonary function tests performed before lung biopsy were available in 15 of 17 patients. A restrictive ventilatory defect was found in 10 of 15 patients. A single patient (patient number six, never-smoker) had a mild obstructive defect (forced expiratory volume in one second/forced vital capacity $68 \%$ ). Lung volumes were normal in four patients. A decrease of transfer factor for carbon monoxide was present in all 12 tested patients, while transfer coefficient was lowered in only four of 11 patients. Ten patients were hypoxaemic at rest (arterial oxygen tension $\left.\left(\mathrm{Pa}, \mathrm{O}_{2}\right)<10 \mathrm{kPa}\right)$. At exercise, $\mathrm{Pa}_{2} \mathrm{O}_{2}$ decreased (mean variation of $-1.3 \pm 0.85 \mathrm{kPa}$ ) and was $<10 \mathrm{kPa}$ in the 10 tested patients. Alveolar-arterial oxygen $\left(\mathrm{O}_{2}\right)$ difference $\left(100 \% \mathrm{O}_{2}\right)$ was in the normal range in the five tested patients.

\section{Computed tomography of the chest}

CT images were available for review in all cases, with $1.0-1.5 \mathrm{~mm}$ collimation images in 16 of 17 cases. Diffuse infiltrative opacities were present in all cases. Reticular and ground glass opacities were the most frequent findings. Honeycomb cysts were not present. The main finding for each patient was represented by areas of consolidation in six of 17 cases, ground glass attenuation in five of 17 , reticular opacities in five of 17 , and peribronchovascular thickening in one of 17. When present, areas of air-space consolidation were disseminated, small-sized, with a predominantly peribronchovascular distribution, except in patient number 17 , who had large areas of postero-basal consolidation on initial CT.

A diagnostic pattern of UIP (i.e. a combination of reticular opacities, traction bronchiectasis and honeycomb cysts, in the absence of prominent ground glass attenuation or consolidation) was not found in any case, although this diagnosis was eventually considered the most likely in the two cases pathologically diagnosed with UIP. None of the cases with a pathological diagnosis of NSIP had CT findings suggestive of UIP; in 10 of 11 cases, CT findings included a combination of ground glass opacities, intralobular reticular opacities, irregular linear opacities, and traction bronchiectasis, compatible with published descriptions of NSIP [8, 15-18]. CT findings were considered more suggestive of organising pneumonia in the remaining case of NSIP. CT features did not differ significantly between the 11 patients with a pathological NSIP pattern and the six patients with a nonNSIP pathological pattern (Chi-squared analysis, data not shown)

\section{Biology}

Bronchoalveolar lavage was performed in 14 patients. The differential cell pattern was lymphocytic (lymphocytes $>25 \%$ ) in seven patients $(50 \%)$, including four patients with NSIP, one with lymphoid interstitial pneumonia, one with organising pneumonia, and one with UIP. A neutrophilic cell pattern (neutrophils $>10 \%$ ) was found in three patients with NSIP. The cell pattern was mixed (lymphocytes $>25 \%$, neutrophils $>10 \%$ ) in two patients with NSIP, predominantly eosinophilic $(45 \%)$ in patient number 15 who had UIP, and normal in patient number 13 .

Antinuclear antibodies were present in nine of 15 patients (table 1), with a median titre of one of 256, and a homogenous pattern in three cases. Anti-tRNA-synthetase cytoplasmic antibodies were present in seven patients, including anti-Jo-1 and anti-PL7 antibodies; other antitransfer ribonucleic acid (tRNA)-synthetase antibodies (anti-PL12, -OJ, -EJ) and anti-cytoplasmic antibodies (anti$\mathrm{KJ}$, -Mas) were negative. Antineutrophil cytoplasmic antibodies were negative in all 12 patients studied. Laboratory tests did not differ significantly between patients with respiratory or cutaneous/muscular symptoms as the initial manifestation of the disease.

\section{Treatment and outcome}

All patients (except patient number nine) were treated with corticosteroids, with a daily dose of $\sim 1 \mathrm{mg} \cdot \mathrm{kg}^{-1}$ prednisone or prednisolone orally in most patients. Twelve patients subsequently received immunosuppressive agents, including azathioprine $(n=7)$, cyclophosphamide $(n=7)$, methotrexate $(n=5)$, cyclosporine A $(n=4)$, chlorambucil $(n=5)$, colchicine $(n=2)$, and hydroxychloroquine $(n=1)$. In addition, one patient was treated with plasma exchanges, and five patients received intravenous polyclonal $\gamma$-globulins as a treatment of DPM (table 1).

The median length of follow-up after the pathological diagnosis was $3.4 \mathrm{yrs}$ (range, 0.2-1.8 yrs), and the median time interval from the onset of pulmonary manifestations to the last follow-up (or death) was 3.5 yrs (ranging 145 days12.3 yrs). Twelve patients were alive at last follow-up. Five patients died and one underwent pulmonary transplantation over that period of time, with a median time interval from lung biopsy to death of 3.5 yrs. Survival at 1 -yr was $100 \%$, 2-yr survival was $92.8 \%$, and 5 -yr survival was $50 \% ; 77 \%$ of the patients were alive without having received a lung transplant 3 yrs after biopsy, and $44 \%$ after 5 yrs. Survival at 3-yrs did not differ significantly between patients with NSIP $(89 \%)$ and patients with a non-NSIP diagnosis $(80 \%)$. 
Cause of death was progressive interstitial lung disease leading to respiratory failure in all cases. Among the six patients who died or underwent pulmonary transplantation, four had a histopathological diagnosis of NSIP (including one with cellular NSIP), one was diagnosed with lymphoid interstitial pneumonia, and one with unclassifiable interstitial pneumonia. The poor outcome in these patients could not be correlated with any specific clinical or radiological features at the time of diagnosis. A poor outcome was found in four of 10 patients with dermatomyositis, one of four patients with polymyositis, and none of three patients with amyopathic dermatomyositis.

The remaining 11 patients improved with treatment according to clinical, radiological and lung function criteria. Improvement was complete in two patients, and partial in nine with persistence of moderate functional and/or radiological sequelae. Five episodes of relapse of the lung disease occurred while decreasing the treatment in three of 12 patients. Increasing the corticosteroid treatment and/or adding immunosuppressive therapy allowed clinical recovery of the pulmonary relapse.

Outcome of the muscular disease with treatment was as follows: complete or partial clinical recovery in eight of 14 patients, stable disease in three, and worsening despite treatment in three patients. Skin manifestations resolved or improved with treatment in 11 of 13 patients, and worsened in two patients.

\section{Discussion}

NSIP was found to be the most common histological pattern of interstitial pneumonia (65\% of cases), particularly in patients with amyopathic dermatomyositis, and in patients with the interstitial pneumonia preceding the onset of cutaneous and/or muscular manifestations.

Amyopathic dermatomyositis has been individualised in the spectrum of DPM $[11,19]$. In most patients, amyopathic dermatomyositis does not progress to myositis [20], a finding which may reflect the effect of early treatment [11], or indicating that it may be a distinct entity in the spectrum of DPM.

Since most studies on DPM-associated lung disease required muscle disease as a mandatory criterion for the diagnosis of DPM and therefore did not include patients with amyopathic dermatomyositis, little is known about pulmonary manifestations of this "forme fruste" of connective tissue disease. In the initial description of six cases [19], one patient had "pulmonary fibrosis", which persisted despite corticosteroid treatment. Lung involvement may be uncommon in amyopathic dermatomyositis, since no case of interstitial lung disease was reported in two recent series of 37 and 13 patients, respectively [20, 21]. In the present study, three patients presented with amyopathic dermatomyositis. All three had an overall follow-up of at least 2 yrs ( $>6$ yrs in patient number one), with no evidence of clinical muscle manifestations, consistent with diagnostic criteria [11]. Histopathological analysis demonstrated NSIP in all three patients. Diffuse alveolar damage has also been reported in three patients with amyopathic dermatomyositis and acute respiratory failure [6]. Of note, the possibility of interstitial lung disease in patients with amyopathic dermatomyositis parallels the occurrence of interstitial lung disease in patients with systemic sclerosis sine scleroderma. Thus, limited forms of connective tissue diseases should be systematically considered in patients with interstitial lung disease, particularly with a histological pattern of NSIP.

Manifestations of connective tissue diseases may be initially limited to a single organ, so that interstitial lung disease may precede the inflammatory muscle or skin disease, as in four patients in the current study $[3,4,22]$. The time interval between respiratory and muscular or skin symptoms was clinically relevant, with a median delay of 100 and 340 days, respectively. When a NSIP pattern is found on lung biopsy, attention should be paid to muscular or cutaneous symptoms that may appear at some time during the course of the disease $[11,19]$, particularly in patients with anti-tRNA-synthetase antibodies. Lung involvement may be frequently associated with cutaneous manifestations of DPM, since 13 of 17 of patients from the current study had skin manifestations, whereas dermatomyositis accounted for a minority of the cases in a large series of DPM [23].

Early reports of the interstitial lung disease associated with DPM suggested similarities with idiopathic pulmonary fibrosis [3-5, 24], but it is likely that some of these cases may actually correspond to fibrosing NSIP, according to the current pathological criteria. Cellular interstitial pneumonia was also described [24], and may also well correspond to NSIP. A recent study by Douglas et al. [1] of 22 patients with DPM who underwent surgical lung biopsy concluded that the interstitial lung disease associated with DPM was characterised by a pathological NSIP pattern in $82 \%$ of cases. NSIP was present in four of 11 cases in another study, with UIP in five of 11 cases [12]. In addition to NSIP and UIP, other histopathological patterns have been described in patients with DPM, including diffuse alveolar damage [1, 5], organising pneumonia $[1,4,5,12$, and the present study], and desquamative interstitial pneumonia [25].

Since only cases with lung biopsy were included in the present study, some selection bias cannot be excluded. Namely, it is conceivable that patients with clinical and/or radiological findings not typical of UIP may have been offered lung biopsy more often than those with typical CT findings. As a consequence, the prevalence of NSIP among patients with DPM-associated interstitial lung disease may be overestimated. However, five patients underwent lung biopsy before the individualisation of NSIP, four of which were diagnosed as having NSIP. Thus, the authors believe that a selection bias is unlikely in the present histopathological series.

Interestingly, a high prevalence of NSIP has also been found in histopathological series of interstitial pneumonia associated with other connective tissue diseases, particularly systemic sclerosis [26]. NSIP was found in $77 \%$ of patients with systemic sclerosis-associated interstitial lung disease, whereas UIP accounted for only $7 \%$ of the cases [27]. NSIP has also been described in rheumatoid arthritis, primary Sjögren's syndrome, and systemic lupus erythematosus [7, 8].

In conclusion, nonspecific interstitial pneumonia was found to be the most common histological pattern of interstitial pneumonia encountered in dermatomyositis/polymyositis, particularly in patients with amyopathic dermatomyositis and in dermatomyositis/polymyositis patients with respiratory symptoms as the initial clinical manifestation of the connective tissue disease. The clinician should remain alert to potential muscular or cutaneous symptoms and "formes frustes" of connective tissue disease whenever a pathological diagnosis of nonspecific interstitial pneumonia is made.

Acknowledgements. The authors would like to thank R. Frognier and V. Leigh for assistance in data collection, and S. Barrot for data entry and secretarial work, and L. Thomas for clinical advice in several patients.

The following members of the GERM"O"P participated in the study by including one or more cases: J. Boyer (Vienne), J. Cadranel (Paris), 
P. Camus (Dijon), J.F. Cordier (Lyon), I. CourtFortune (Saint-Etienne), B. Crestani (Paris), P. Delaval (Rennes), G. Devouassoux (Grenoble), G. Mangiapan (Créteil), M. Reynaud-Gaubert (Marseille), B. Wallaert (Lille).

The following pathologists participated in the study by reviewing one or more cases: E. Brambilla (Grenoble), F. Capron (Paris), L. Chalabreysse (Lyon), R. Loire (Lyon), F. Piard (Dijon), F. Thivolet-Béjui (Lyon).

\section{References}

1. Douglas WW, Tazelaar HD, Hartman TE, et al. Polymyositis-dermatomyositis-associated interstitial lung disease. $\mathrm{Am}$ J Respir Crit Care Med 2001; 164: 1182-1185.

2. Marie I, Hachulla E, Hatron PY, et al. Polymyositis and dermatomyositis: short term and long term outcome, and predictive factors of prognosis. $J$ Rheumatol 2001; 28: 2230 2237.

3. Duncan PE, Griffin JP, Garcia A, Kaplan SB. Fibrosing alveolitis in polymyositis. A review of histologically confirmed cases. Am J Med 1974; 57: 621-626.

4. Schwarz MI, Matthay RA, Sahn SA, Stanford RE, Marmorstein BL, Scheinhorn DJ. Interstitial lung disease in polymyositis and dermatomyositis: analysis of six cases and review of the literature. Medicine (Baltimore) 1976; 55: 89-104.

5. Tazelaar HD, Viggiano RW, Pickersgill J, Colby TV. Interstitial lung disease in polymyositis and dermatomyositis. Clinical features and prognosis as correlated with histologic findings. Am Rev Respir Dis 1990; 141: 727-733.

6. Lee CS, Chen TL, Tzen CY, et al. Idiopathic inflammatory myopathy with diffuse alveolar damage. Clin Rheumatol 2002; 21: 391-396.

7. Katzenstein ALA, Fiorelli RF. Nonspecific interstitial pneumonia/fibrosis. Histological features and clinical significance. Am J Surg Pathol 1994; 18: 136-147.

8. Cottin V, Donsbeck AV, Revel D, Loire R, Cordier JF. Nonspecific interstitial pneumonia. Individualization of a clinicopathologic entity in a series of 12 patients. Am J Respir Crit Care Med 1998; 158: 1286-1293.

9. Bohan A, Peter JB. Polymyositis and dermatomyositis (first of two parts). N Engl J Med 1975; 292: 344-347.

10. Euwer RL, Sontheimer RD. Amyopathic dermatomyositis: a review. J Invest Dermatol 1993; 100: Suppl. 1, 124-127.

11. Euwer RL, Sontheimer RD. Amyopathic dermatomyositis (dermatomyositis sine myositis). Presentation of six new cases and review of the literature. J Am Acad Dermatol 1991; 24: 959-966.

12. Marie I, Hachulla E, Cherin P, et al. Interstitial lung disease in polymyositis and dermatomyositis. Arthritis Rheum 2002; 47: 614-622.

13. Austin JH, Muller NL, Friedman PJ, et al. Glossary of terms for CT of the lungs: recommendations of the Nomenclature Committee of the Fleischner Society. Radiology 1996; 200: 327-331.

14. American Thoracic Society/European Respiratory Society International Multidisciplinary Consensus Classification of the Idiopathic Interstitial Pneumonias. Am J Respir Crit Care Med 2002; 165: 277-304.

15. Kim TS, Lee KS, Chung MP, Kwon OJ, Kim TS, Hwang JH. Nonspecific interstitial pneumonia with fibrosis: serial high resolution CT findings with functional correlation. $\mathrm{Am}$ J Roentgenol 1999; 173: 949-953.

16. Johkoh T, Muller NL, Colby TV, et al. Nonspecific interstitial pneumonia: correlation between thin-section CT findings and pathologic subgroups in 55 patients. Radiology 2002; 225: 199-204.

17. Nishiyama $\mathrm{O}$, Kondoh $\mathrm{Y}$, Taniguchi $\mathrm{H}$, et al. Serial high resolution $\mathrm{CT}$ findings in nonspecific interstitial pneumonia/ fibrosis. J Comput Assist Tomogr 2000; 24: 41-46.

18. Hartman TE, Swensen SJ, Hansell DM, et al. Nonspecific interstitial pneumonia: variable appearance at high-resolution chest CT. Radiology 2000; 217: 701-705.

19. Krain L. Dermatomyositis in six patients without initial muscle involvement. Arch Dermatol 1975; 111: 241-245.

20. el-Azhary RA, Pakzad SY. Amyopathic dermatomyositis: retrospective review of 37 cases. J Am Acad Dermatol 2002; 46: $560-565$.

21. Caproni M, Cardinali C, Parodi A, et al. Amyopathic dermatomyositis: a review by the Italian Group of Immunodermatology. Arch Dermatol 2002; 138: 23-27.

22. Schwarz MI. The lung in polymyositis. Clin Chest Med 1998; 19: 701-712.

23. Hochberg MC, Feldman D, Stevens MB. Adult onset polymyositis/dermatomyositis: an analysis of clinical and laboratory features and survival in 76 patients with a review of the literature. Semin Arthritis Rheum 1986; 15: 168-178.

24. Lakhanpal S, Lie JT, Conn DL, Martin WJ 2nd. Pulmonary disease in polymyositis/dermatomyositis: a clinicopathological analysis of 65 autopsy cases. Ann Rheum Dis 1987; 46: $23-29$

25. Grau JM, Miro O, Pedrol E, et al. Interstitial lung disease related to dermatomyositis. Comparative study with patients without lung involvement. J Rheumatol 1996; 23: 1921-1926.

26. Kim DS, Yoo B, Lee JS, et al. The major histopathologic pattern of pulmonary fibrosis in scleroderma is nonspecific interstitial pneumonia. Sarcoidosis Vasc Diffuse Lung Dis 2002; 19: 121-127.

27. Bouros D, Wells AU, Nicholson AG, et al. Histopathologic subsets of fibrosing alveolitis in patients with systemic sclerosis and their relationship to outcome. Am J Respir Crit Care Med 2002; 165: 1581-1586. 